\title{
Expert System Based on an Ontology Method to Analyze Types of Arabica Coffee Beans
}

\author{
https://doi.org/10.3991/ijes.v5i2.6908 \\ Michelle Angelica \\ University of Multimedia Nusantara, Indonesia \\ michelle.angelica@student.umn.ac.id \\ Friska Natalia Ferdinand \\ University of Multimedia Nusantara, Indonesia \\ friska.natalia@umn.ac.id
}

\begin{abstract}
In the past few decades, the use of ontologies in information systems has become popular in many fields, such as website development, database integration, and natural language processing. Because many kinds of coffee beans can be used in coffee shops, the prospective coffee house entrepreneur meets obstacles in terms of choosing the right coffee beans because of multiple unique characteristics. In order to help this cohort make decisions, our study proposed a simulation ontology-based matching for coffee bean selection by inserting three parameters - aroma, flavor, and sour level — as inputs on the website. Arabica coffee bean is used as the principal object in this study and the outputs would be the beans matched with the parameters that had been inserted. In this study, the system model gained from the ontology method is shown in the implementation by using an example.
\end{abstract}

Keywords-Arabica coffee beans, Ontology, OWL, Protégé, SPARQL

\section{Introduction}

Coffee is a type of beverage that is a daily need for people nowadays, and unconsciously it has become a trend or lifestyle for Indonesian people. Indonesia itself has played a major role in coffee consumption. The country was ranked the third biggest coffee producer in the world after Brazil and Vietnam. In the early 2000s, the popularity of coffee in Indonesia rose unexpectedly because of American coffee chains such as Starbucks and The Coffee Bean \& Tea Leaf opening their first branches there. Since 2013, many local coffee shops have been owned by Indonesian entrepreneurs. They are based on the tendencies and lifestyles of coffee drinkers.

In computer science and information science, an ontology is described as a structured set of terms and concepts representing the meaning of information domains, whether in the metadata of a namespace or the elements of a knowledge domain. Ontology in the field of computer and information science refers to artifact techniques based on specialized vocabulary used to refer to a reality, plus a set of explicit as- 
sumptions relating to the intended meaning of the vocabulary [2]. Bellur and Kulkarni proposed a more exhaustive matchmaking algorithm based on the concept of matching bipartite graphs [8]. Gao et al. proposed an ontology-based semantic matchmaking method [4]. This approach shows more generality rather than domain-specific compared with aforementioned research. In order to match, a user query for the matching process is semantically matched with a set of existing service providers to present reasoning framework design combining ontology and rules [3].

The purpose of this study was to the software implementation driven ontologies provide conceptual schemata whose main focus is normally on data storage and data manipulation and is utilized to software development activities, with the goal of guaranteeing data consistency. The system model based on the ontology method uses different kinds of coffee beans, which can help entrepreneurs to open coffee shops. An analysis on coffee beans in this study uses the ontology method.

\section{$2 \quad$ Literature Review}

This section is available for background to the topics in this paper. First, the overview supply partner matching is briefly covered. Second, we deal with the basic idea of ontology area. Next, the system development life cycle is shown. In the earliest literature, "fuzzy ontology"-based supply partner matching research found the right supplier in the supply chain network by utilizing a matching system, which was made based on fuzzy ontology [3]. The ontology matching system was used to identify potential suppliers or partners and picked a partner or supplier that had the biggest imaginable level of all. Subsequent literature shows the ontology for the description of drug discovery investigations with existing ontologies was combined to form a new ontology called drug discovery investigations (DDIs). Data contained in DDIs can be transferred and promoted along with the increase of information. The purpose of this research is from DDIs are to be expected to be found different types of drugs or vaccines that can cure the incurable diseases at present [5]. The extant literature describes information technology project system development life cycles by using the comparative study described in the system development life cycles (SDLC) system. Eight kinds of SDLC were discussed in this research: Waterfall, Incremental, V Model, B Model, Fountain Model, Prototyping, Structured Systems Analysis, and Design Methods. The purpose of this research is to specifically explain each kind of the SDLC for better understanding [1]. On the basis of these previous studies, this research developed an application based on the Protégé, JESS, and SWRL platforms. The ontology method was used to classify coffee beans with these data properties: hasAromaName, hasCoffeeBeansName, hasDoseName, hasFlavourName, and hasSourLevelName. The results of this study can be described by an application that can help the coffee shop entrepreneurs effectively in terms of gathering the information and time used to collect information required to open up a coffee shop. All the coffee beans were selected based on the components that have been saved to form an OWL data type. 


\section{Problem Definition and Solution Procedure}

In this research, the Arabica coffee bean is used as the type. All the characteristics of the type and composition of the coffee dose used for making coffee-based on beverages type. Interviews regarding data collection techniques were conducted with the owners of coffee shops and roasters. Two coffee houses and three roasters were taken for research objects. The monastery is an area where the coffee beans are roasted and processed. Roasting is the creation stage of the coffee aroma, and there is a distinct flavor of the coffee beans with heat treatment. Coffee beans naturally contain abundant organic compounds that form a distinctive flavor and aroma. Arabica coffee beans are used as the principal object in this research, and the dose measurement, composition and the characteristics of the Arabica coffee beans are shown in Tables 1 to 3 .

OntoGraf is one of the features found in software protégé since version 4.1. The function of OntoGraf is to provide all users of the protégé with the ability to interactively navigate the relations contained in the file ontology OWL (web ontology language). A wide variety of layouts given in this menu allow the user to freely organize protégé file structure ontology relations that are supported in OntoGraf, including subclass, individual, and object properties. Figure 1 shows a file OntoGraf in ontology

Table 1. Kinds of coffee beans and dose measurement

\begin{tabular}{|l|c|c|}
\hline $\begin{array}{c}\text { Kinds of Arabica Coffee } \\
\text { Beans }\end{array}$ & $\begin{array}{c}\text { Asagao Coffee Dose Measurement } \\
(\mathbf{1} \text { shot }=\mathbf{3 0} \mathbf{~ m L})\end{array}$ & $\begin{array}{c}\text { Koultoura Coffee Dose Meas- } \\
\text { urement (1 shot = 30 } \mathbf{~ m})\end{array}$ \\
\hline Aceh Gayo & 1 shot & - \\
\hline Jawa Preanger & 2 shot & - \\
\hline Bali Kintamani & 2 shot & - \\
\hline Toraja Sapan & 2 shot & - \\
\hline Mandheling Danau Toba & 2 shot & 1 shot \\
\hline Brazil & - & 1 shot \\
\hline Kolombia & - & 2 shot \\
\hline Solok Selatan & - & \\
\hline
\end{tabular}

Table 2. Kinds of coffee beverages and compositions

\begin{tabular}{|l|c|c|}
\hline $\begin{array}{c}\text { Kinds of Arabica Coffee } \\
\text { Beans }\end{array}$ & $\begin{array}{c}\text { Asagao Coffee Composition } \\
\text { (Coffee: Milk) }\end{array}$ & $\begin{array}{c}\text { Koultoura Coffee Composition } \\
\text { (Coffee: Milk) }\end{array}$ \\
\hline Latte & $1: 5$ & $2: 3$ \\
\hline Cappuccino & $1: 2$ & $1: 2$ \\
\hline Flat White & $2: 5$ & $2: 5$ \\
\hline Picollo & $2: 3$ & $2: 3$ \\
\hline Macchiato & $1: 1$ & $2: 2$ \\
\hline Americano & $2: 5$ & $2: 5$ \\
\hline
\end{tabular}


Table 3. Kinds of coffee beans and dose measurement

\begin{tabular}{|l|c|c|c|}
\hline $\begin{array}{c}\text { Kinds of Arabica Coffee } \\
\text { Beans }\end{array}$ & Flavor & Aroma & Sour Level \\
\hline Aceh Gayo & Full Body & Fruit Tendency & Low Sour \\
\hline Bali Kintamani & Medium Body & Fruit Tendency & High Sour \\
\hline Toraja Sapan & Medium Body & Nut Tendency & Low Sour \\
\hline Flores Bajawa & Full Body & Fruit Tendency & High Sour \\
\hline Sunda Hejo & Medium Body & Herb Tendency & Low Sour \\
\hline Lintong & Medium Body & Herb Tendency & Low Sour \\
\hline Java Preanger & Medium Body & Nut Tendency & High Sour \\
\hline Papua Wamena & Medium Body & Fruit Tendency & High Sour \\
\hline
\end{tabular}

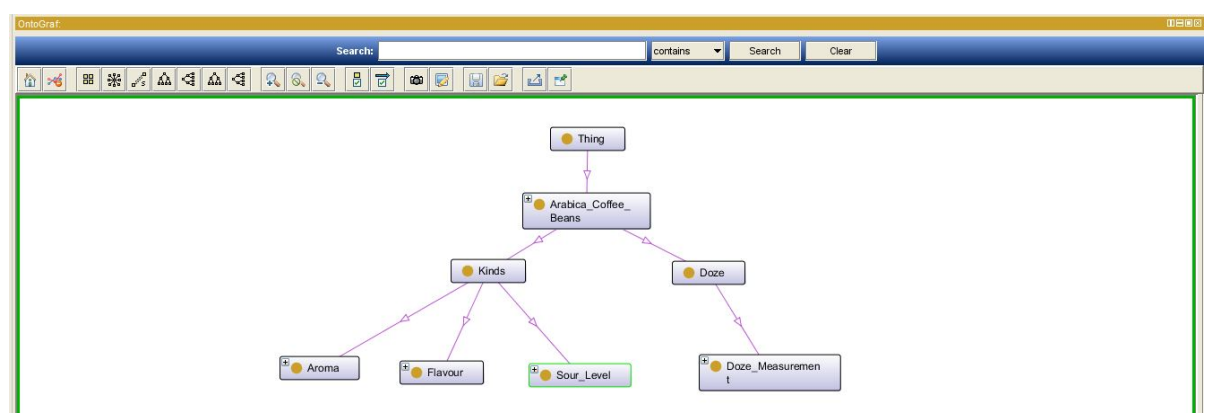

Fig. 1. Classes and subclasses

language (OWL). In an ontology model, there are three main components, which are class and subclass, object property, and data type property. The results of these components that have been saved will form an OWL data type. The following is an explanation of the three main components used in this research.

\subsection{Class and subclass definition}

Class is the primary point of an ontology and the explained of the concept contained in a domain. The domain comprises multiple instances or individuals. A class can have subclasses that are used to explain more specific objects shown in Figure 2. OWL defines the root of all things that exist with the "owl: Thing". All classes are created implicitly a subclass of "owl: Thing". Making classes uses "owl: Class" and declares the subclass with "RDFS: subClassOf".

One core class exists, Arabica Coffee Beans. Two other classes are Kinds and Dose. Class "Kinds" has three subclasses: Aroma, Flavor, and Sour Level. Meanwhile, class "dose" has one subclass, which is dose measured. Figure 3 shows the classes and subclasses that were inserted in the protégé software. Figure 4 shows the ontology model created by using the protégé software as a whole. OntoGraf results from OWL files have been created. Figure 5 describes the relation of Arabica coffee beans in an ontology model with the subclasses. 


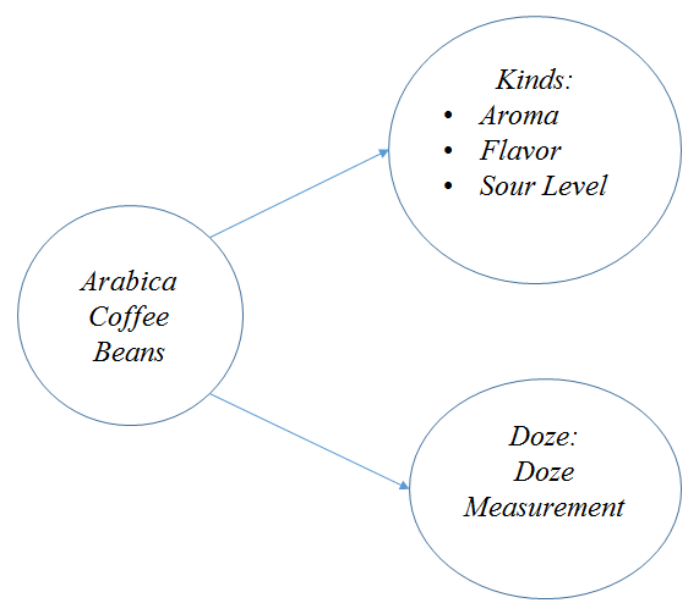

Fig. 2. Classes and subclasses

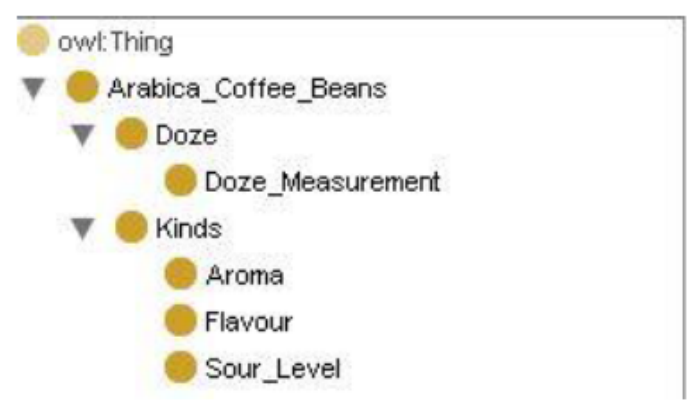

Fig. 3. Classes and subclasses

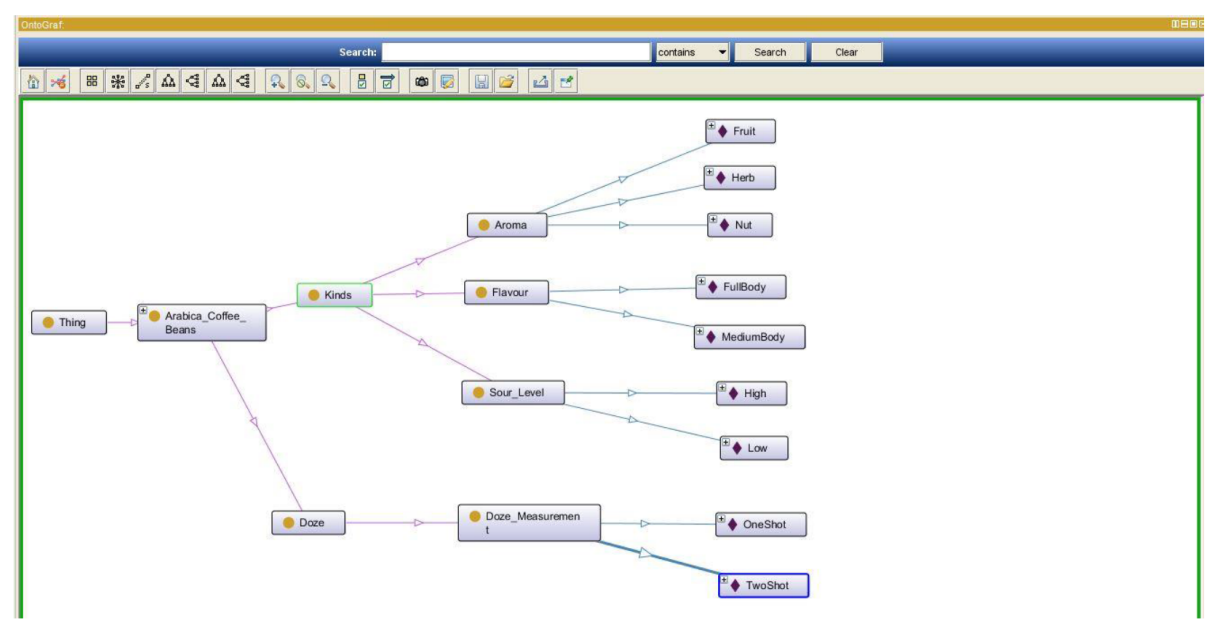

Fig. 4. Ontology model of classes and subclasses 


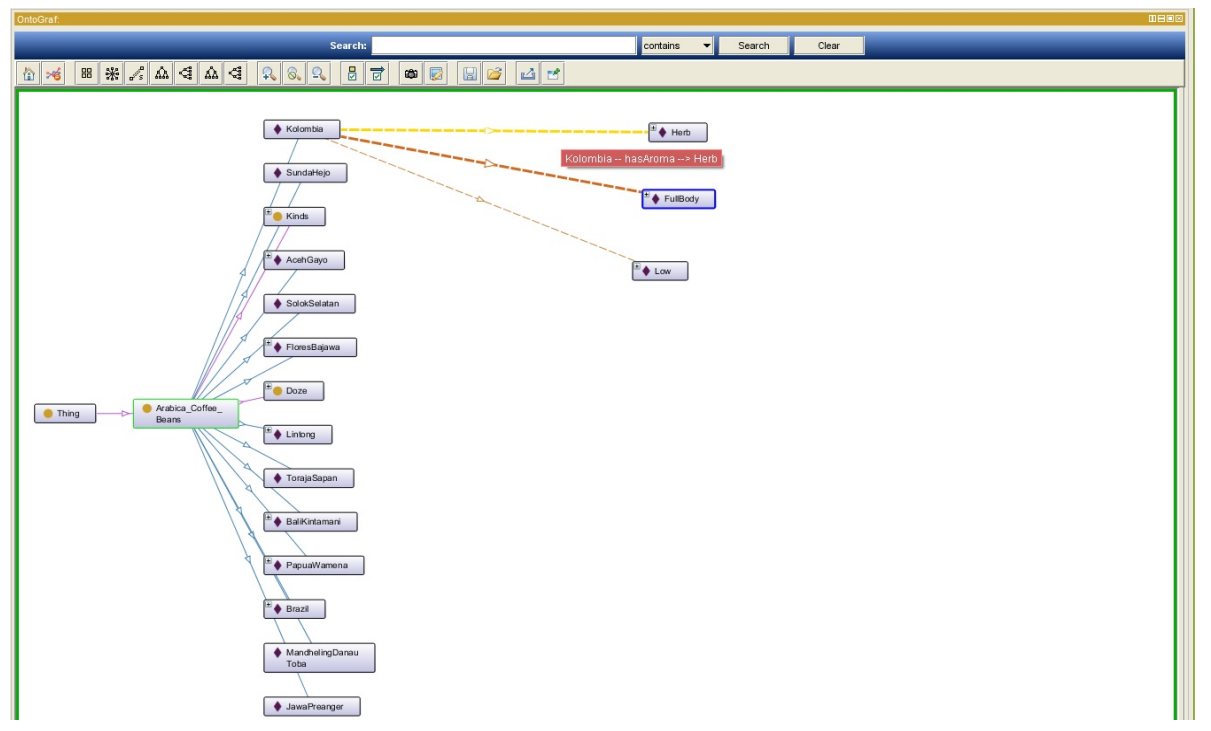

Fig. 5. Ontology model of classes and subclasses

\subsection{Object property definitions}

Several characteristics of the type of object properties follow [2]:

- Functional Properties: These characteristics state that an individual is only connected to one other individual. Another name for these characteristics are single-valued properties.

- Inverse Functional Properties: These features are the inverse of the characteristic functional properties. However, they still have the characteristics that an individual remains only relate to one another individual.

- Transitive Properties: An individual A connects to individual B, and individual B is connected with individual $\mathrm{C}$. It can be concluded that individual A relates to individual $\mathrm{C}$ through the existing property.

- Symmetric Properties: An individual A connects to individual B but also connects the individual and individual $\mathrm{A}$.

In this study, there are two principal object properties, hasDose and hasKinds. The "hasDose" object property has one sub-property called hasDose_Measurement, and the "hasKinds" object property has three sub-properties called hasAroma, hasFlavour, and hasSourLevel. These object properties connect all of Kinds and Dose subclasses to Arabica Coffee Beans class. Figure 6 shows the object properties that were made by using protégé software. In this part, the modeling protégé and OWL file are the results of modeling using data that have been obtained in a software-acquired protégé ontology modeling graph. The modeling ontology graph illustrates the relationship of data that have been obtained as a whole [6]. Having described the overall relationship of the data that have been obtained, the next step is to make a file of 
OWL using software protégé. File OWL functions as a variable storage file with the value of the variable, making it into a kind of database for the method ontology. OWL defines the root of all things that exist with the "Owl: Thing". All classes are created implicitly as a subclass of "Owl: Thing". For example, create classes using "Owl: Class" and declare the subclass with "RDFS: subClassOf".

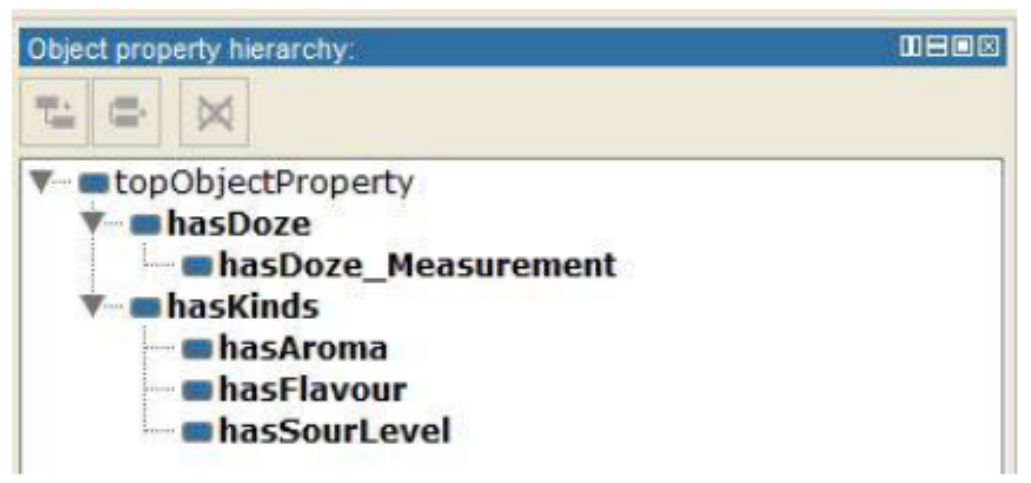

Fig. 6. Object properties

\subsection{Datatype property definitions}

It is the same with classes that can be written in a hierarchical property. They can also be expressed as "subPropertyOf" with "RDFS: subPropertyOf". To provide limits to a property, they can use "RDFS: domain" and "RDFS: range," which is also called the global restriction as it applies generally and is not confined to a particular class.

In this research, five data type properties were used: hasAromaName, hasCoffeeBeansName, hasDoseName, hasFlavourName, and hasSourLevelName. Figure 7 shows these properties were made by using protégé software. Table 4 shows details about each type in the data type properties.

Figure 8 shows that 12 blue arrows pointing from the class "Arabica coffee beans" are individuals of that class. The subclass is marked with 3 pink arrows lines.

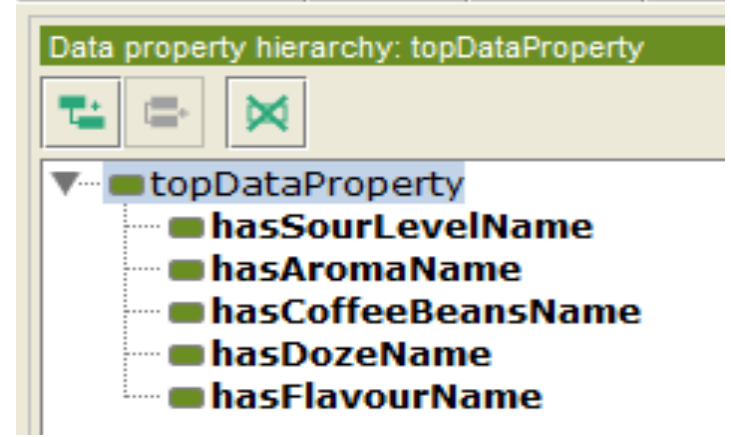

Fig. 7. Data type properties 


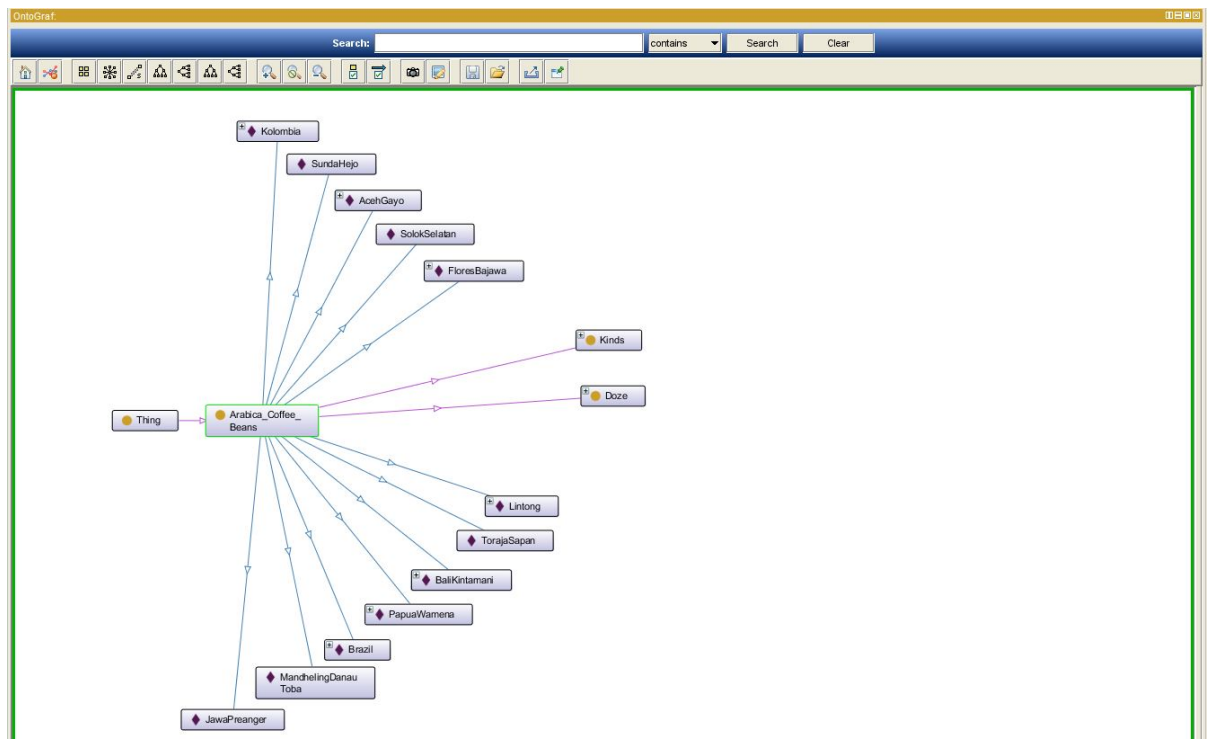

Fig. 8. Individual class “Arabica Coffee Beans" in OntoGraf

Table 4. Range and type of datatype properties

\begin{tabular}{|l|l|l|}
\hline \multicolumn{1}{|c|}{ Property } & Range & Type \\
\hline hasAromaName & String & Datatype Property \\
\hline hasCoffeeBeansName & String & Datatype Property \\
\hline hasDoseName & String & Datatype Property \\
\hline hasFlavourName & String & Datatype Property \\
\hline hasSourLevelName & String & Datatype Property \\
\hline
\end{tabular}

\section{$4 \quad$ Implementation and Results}

The implementation of the ontology method is a system of selection types of coffee beans that have been made previously using the file OWL and a SPARQL query. In this application, the user will be asked to enter the input of three predefined values in the drop-down list options. The parameters are aroma, flavor, and sour level. Figure 9 shows the code used to perform a SPARQL query on the coffee beans electoral system. All data of all variables that exist within the clause "WHERE" were taken by using the phrase "SELECT DISTINCT*". The function "FILTER" was needed to sift through all the data returned. Filtering was done by comparing the value of these variables: ?AromaName, ?FlavourName, and ?SourName. The value of all these variables is matched with the key words that the user entered and is saved in the variables: \$aroma, \$flavor, and \$sour. The query sentence that was made was run using the existing functionality within the library, a SPARQL Query RAP.

Figure 10 is a screenshot of the final appearance for the application on the website, which was taken using a mobile phone browser. If users typed or clicked on the menu 
button (located on the upper right side of the page), the menu bar displayed. Figure 10 also shows the appearance of the application after users typed or clicked on the menu bar. The application's functions are to select coffee beans and help potential coffee shop entrepreneurs efficiently gather and save time to collect information. In order to make the website search for a type of coffee bean, there a few steps to build it:

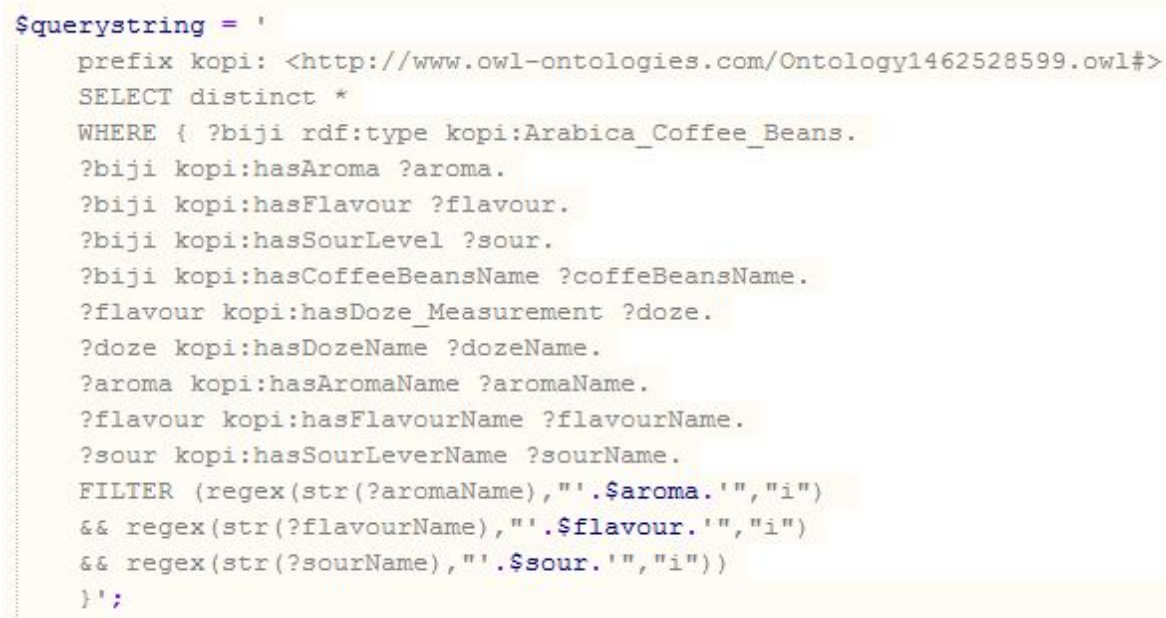

Fig. 9. Query code

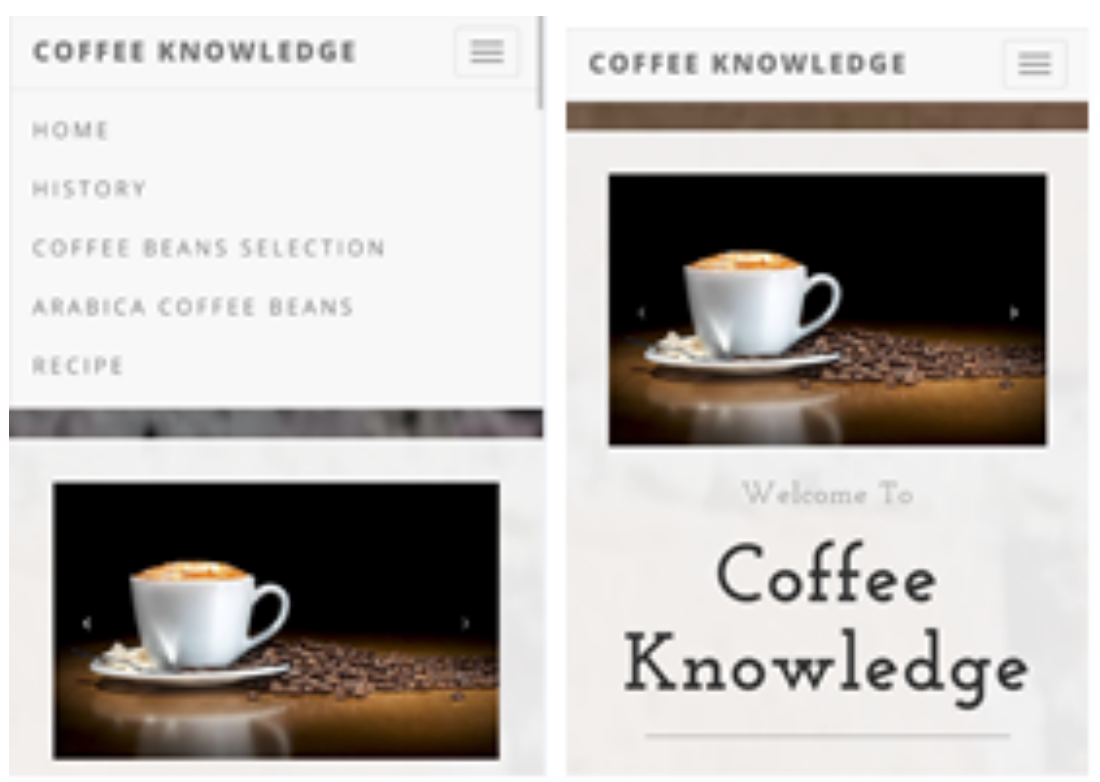

Fig. 10.Application menu 
Step 1: Modeling protégé OWL \& File. Data have been obtained on software acquired protégé ontology modeling graph. Modeling ontology graph illustrates the relationship of data that have been obtained as a whole.

Step 2: Described the overall relationship of the data that has been obtained and continue by making file OWL using software protégé. File Web ontology language (OWL) to function as a variable storage file with the value of the variable, making it into a kind of database for the method ontology.

Step 3: A SPARQL query (an acronym for a SPARQL Protocol and RDF Query Language) is created in advance. A SPARQL query serves to attract and manipulate the data that have been stored in the format of the Resource Description Framework (RDF), a Web ontology language (OWL).

Step 4: The next step is to run a SPARQL query that has been made on the platform PHP (Hypertext Preprocessor) using RDF API library. This is done to be able to display the systems that have been created using file Web ontology language (OWL) and a SPARQL query on the site.

Step 5: After steps 1-4 have been completed, the website can be created as an expert system based on the ontology method.

On the basis of this result, users are becoming more aware of the kinds and origins of coffee beans consumed by society by the existence of "Arabica Coffee Beans". In addition, users can search for coffee beans of their preferences using the given parameters on the "Coffee Beans Selection" menu to make search process more effective and efficient based on the difference of dose ratio or dose measurement that was used for each different kind of coffee beans. The result is shown in Figure 11.

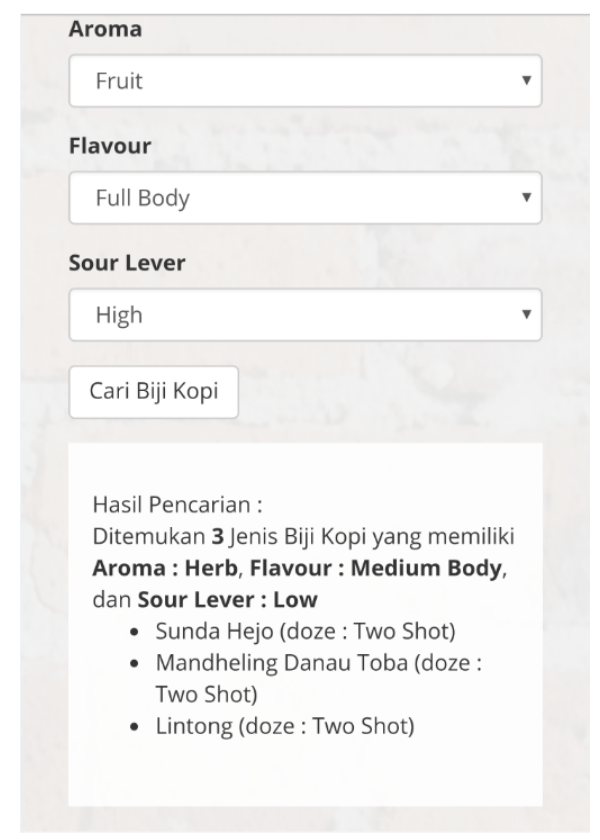

Fig. 11.Result 


\section{Conclusions}

This research was concluded using the ontology method, and the application was made as an output of this research. In order to categorize the type of coffee beans with one another can be made by finding a parameter that serves as a separator between one type of coffee beans with coffee beans more in development. In this study, three parameters were used to categorize the various types of coffee beans: aroma, flavor, and sour level. The system model gained from the ontology method is shown in the implementation by using an example. For future research, the decision parameter scope can be expanded to reach a more complex issue and find a better solution procedure.

\section{References}

[1] Abu - Taieh, E.M.O., El Sheikh, A.A., Abu - Tayeh, J.M., El - Mahied, M.T. (2011). Information Technology Project System Development Life Cycles : Comparative Study.

[2] Guarino, N. (1998). Formal Ontology and Information Systems. In N. Guarino (Ed.), . Formal Ontology in Information Systems.

[3] G. Li, Y. Liu and B. Chen, Ontology and rule combined reasoning framework design. ICIC Express Letters, vol. 4, no. 5(B). pp. 1753-1759, 2010

[4] H. Gao, F. R.Omer J. A. Nick and D. Chen, Ontology-based semantic matchmaking approach, Advance in Engineering software, vol. 38, pp. 56-67, 2007

[5] Lee,H.K., Ferdinand, F.N., Kim, Taioiun. (2011). Fuzzy Ontology - Based Supply Partner Matching, Vol. 5, No. 9, pp: 3329-3334.

[6] Newman, David R. (2010). The Building and Application of a Semantic e- Research Society. The Building and Application of a Semantic e- Research Society.

[7] Qi, Da, King R.D., Hopkins, A.L., Richard, G., Bickerton, J., Larisan,N. (2010). An Ontology for Description of Drug Discovery Investigations.

[8] U. Bellur and R. Kulkarni, Improved matchmaking algorithm for semantic web services based on bipartite graph matching. IEEE International Conference on Web Services. Pp 86-93, 2007. https://doi.org/10.1109/ICWS.2007.105

\section{Authors}

Michelle Angelica is a Student at Universitas Multimedia Nusantara, Department of Information Systems, Nusantara, Indonesia. Young technopreneur with high passion in programing and business development. Have experienced with programing language like php and java.

Friska Natalia Ferdinand is Head of Research Center in Multimedia Nusantara University also Lecturer in Department of Information Systems. She received her $\mathrm{Ph}$.D. in Industrial Engineering from Kyungsung University, Busan, Republic of Korea. Her teaching and research interests include system and analysis design, logistics, e- business and computer programming. She can be reached by e-mail at friska.natalia@umn.ac.id

Article submitted 17 March 2017. Published as resubmitted by the authors 25 April 2017. 